\title{
Effective Collaboration Models for Statisticians and Public Health Departments
}

\author{
Steven E. Rigdon ${ }^{1}$, Elena Naumova ${ }^{2}$, Ian Painter ${ }^{3}$, Yulia Gel ${ }^{4}$ and Howard Burkom5 \\ ${ }^{1}$ Biostatistics, Saint Louis University, St. Louis, MO, USA; ${ }^{2}$ Department of Civil and Environmental Engineering, Tufts University, \\ Medford, MA, USA; ${ }^{3}$ University of Washington, Seattle, WA, USA; ${ }^{4}$ University of Texas, Dallas, TX, USA; 5 Johns Hopkins Applied \\ Physics Laboratory, Laurel, MD, USA
}

\section{Objective}

The session will explore past collaborations between the scientist panelists and public health departments to highlight approaches that have and have not been effective and to recommend effective, sustainable relationship strategies for the mutual advancement of practical disease surveillance and relevant academic research.

\section{Introduction}

Public health departments need enhanced surveillance tools for population monitoring, and external researchers have expertise and methods to provide these tools. However, collaboration with potential solution developers and students in academia, industry, and government has not been sufficiently close or well informed for rapid progress. Many peer-reviewed papers on biosurveillance methods have been published by researchers, but few methods have been adopted in systems used by health departments. In a 2013 BioSense User Group survey with responses from users in more than 40 U.S. states, access to improved analytic methods was a top priority [1]. Among the tools most desired by respondents were the ESSENCE biosurveillance system with multiple analytic tools and statistical software packages such as SAS. Multiple obstacles have slowed the progress of practitioners and developers who seek the development and implementation of useful analytic tools. First, the epidemiological challenges and associated operational constraints are not sufficiently understood among academic developers. Many health departments do not have the resources to hire such developers beyond maintenance of information technology, and the health monitors are typically too busy to publish in peer-reviewed journals. Second, data cannot be shared because of privacy and proprietary limitations with varying local rules. Data-sharing has posed difficult administrative problems, both within and external to health departments, in the course of ISDS Technical Conventions committee efforts to promote interactions through use case problems [2]. Third, aspects of situational awareness vary widely among health monitors at different jurisdictional levels, so analytical challenges and constraints vary widely among potential users. Practitioners have pointed out that "surveillance is local", but local operational and data environments vary widely. A fourth main issue is cross-cultural: Understaffed health departments must respond to successive crises and often lack the time for requirements analysis and technical publication. Such client work situations complicate interaction with academic environments of semester schedules and limited grants and transient student support. This panel brings together academic statisticians who have had successful direct relationships with public health departments to discuss how they have dealt with these challenges.

\section{Description}

Panelists will describe their experiences working on projects for health departments, including actual applications as examples. Discussions will cover system studies as well as the creation and implementation of operational tools. Issues discussed will include:
- Analysis of technical requirements and scoping technical problems for health department utility and tractability of analytic approach.

- Adaptation of traditional statistical approaches, such as relaxation of data distribution assumptions and management of changing data environments

- Communication of solution methods to non-statisticians

- Data-sharing barriers, their effects on progress achieved, and means for overcoming or working around them

- Obtaining and sustaining needed funding for collaborations

Panelists will derive advice for public health practitioners seeking analytical help in the areas of forming relationships, framing problems, communicating results, and seeking funding.

\section{Audience Engagement}

Audience reactions and experiences will be steered to formation of models for collaboration regarding issues of analytic requirements, communication, data-sharing, and funding.

\section{Keywords}

statistical collaboration; analytic methods; Technical Conventions

\section{References}

[1] Gibson J., Karras B.T., Gordon G.S., BioSense 2.0 Governance: Surveying Users and Stakeholders for Continued Development, Online Journal of Public Health Informatics (2014) Vol. 6, No. 1, http://ojphi.org/ojs/index.php/ojphi/article/view/5051

[2] Baer A., Rennick M., Kite-Powell A., Atrubin D., Guidelines for Navigating Human Subjects Review and Preparing Data Sets for Sharing with the ISDS Technical Conventions Committee, Online Journal of Public Health Informatics, Vol 6, No 1 (2014), http://ojphi. org/ojs/index.php/ojphi/article/view/5109

\footnotetext{
*Howard Burkom

E-mail: howard.burkom@jhuapl.edu
} 